\title{
The Self-Made Puzzle: Integrating Self-Assembly and Pattern Formation Under Non-Random Genetic Regulation
}

\author{
René Doursat \\ Institut des Systèmes Complexes, CREA \\ CNRS \& Ecole Polytechnique, Paris \\ http://doursat.free.fr
}

\begin{abstract}
On the one hand, research in self-assembling systems, whether natural or artificial, has traditionally focused on pre-existing components endowed with fixed shapes. Biological development, by contrast, dynamically creates new cells that acquire selective adhesion properties through differentiation induced by their neighborhood. On the other hand, pattern formation phenomena are generally construed as orderly states of activity on top of a continuous 2-D or 3-D substrate. Yet, again, the spontaneous patterning of an organism into domains of gene expression arises within a multicellular medium in perpetual expansion and reshaping. Finally, both phenomena are often thought in terms of stochastic events, whether mixed components that randomly collide in self-assembly, or spots and stripes that occur unpredictably from instabilities in pattern formation. Here too, these notions need significant revision if they are to be extended and applied to embryogenesis. Cells are not randomly mixed but pre-positioned where cell division occurs. Genetic identity domains are not randomly distributed but highly regulated in number and position. In this work, I present a computational model of programmable and reproducible artificial morphogenesis that integrates self-assembly and pattern formation under the control of a nonrandom gene regulatory network. The specialized properties of cells (division, adhesion, migration) are determined by the gene expression domains to which they belong, while at the same time these domains further expand and segment into subdomains due to the self-assembly of specialized cells. Through this model, I also promote a new discipline, embryomorphic engineering to solve the paradox of "meta-designing" decentralized, autonomous systems.
\end{abstract}

\section{Self-Assembly of Pre-Patterned Components}

\subsection{From puzzles to self-assembly}

In the "jigsaw puzzle" metaphor of self-assembling systems, in particular molecular and biological self-assembly, a "piece" of the puzzle represents an elementary component of the system, such as a molecule or a cell. The "shape" of this piece represents its binding affinities with other components - an electric field in the case of molecules (via ionic or hydrogen bonds) or differential adhesion in the case of cells 
(via specific membrane proteins). At any instant, the puzzle finds itself in a certain state, which corresponds to a particular spatial arrangement of its pieces. Associating an energy or cost function with states, the "solutions" of the puzzle can then be defined as the energy minima, i.e., those states where all pieces "fit" well together and satisfy each other's constraints.

Naturally, several fundamental aspects also distinguish complex self-assembling systems from jigsaw puzzles (Fig. 1):

(i) Affinities: The fit between components is not necessarily all-or-none but approximate or flexible (compare Fig. 1a,d) and may exhibit different degrees of well-formedness, associated with varying energy costs. Thus, the "solutions" of the system need not be strict energy minima but simply low-cost states.

(ii) Component types: Components's shapes are far from unique. The system is generally composed of distinct types (molecule species or cell types) shared by a multitude of components that are copies of each other (Fig. 1d-f). This allows for a large number of equivalent states, invariant by permutation of components, and greatly facilitates convergence toward one of the many low-energy solutions.

(iii) Control: No centralized control or "visible hand" actually moves the pieces.
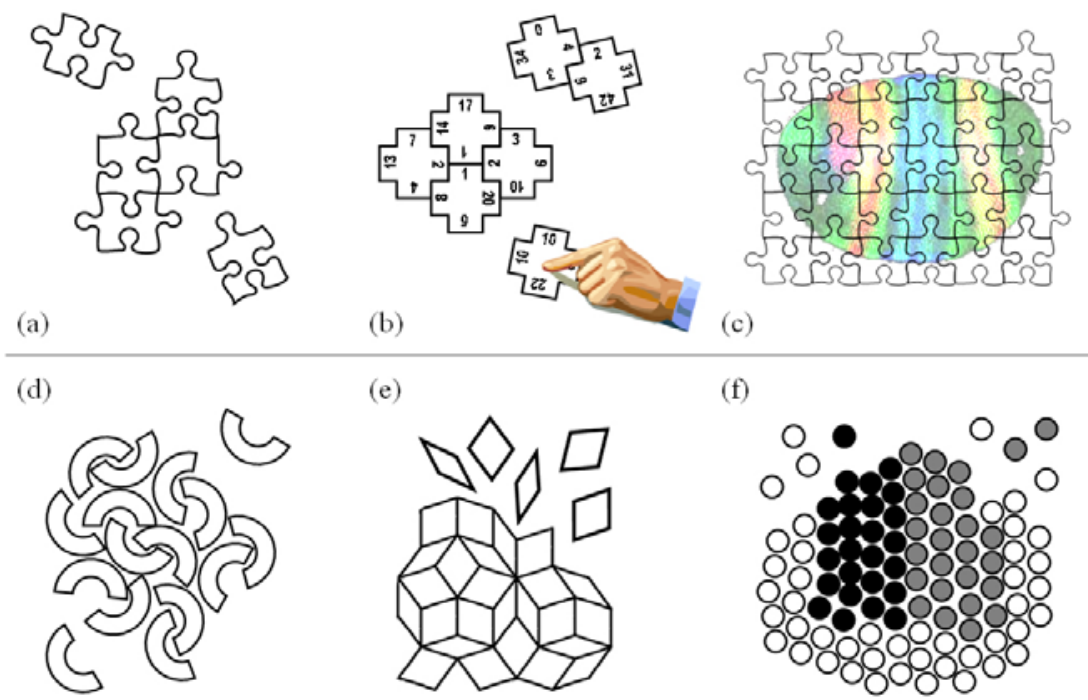

Fig. 1: Differences between jigsaw puzzles and self-assembly. (a)-(c) Jigsaw puzzles are made of uniquely "shaped" pieces, where shape constraints result from specific geometry as in (a), specific markings as in (b) or both geometry and markings as in (c). Compatibility with other pieces is a rare or unique event, and fit between pieces is rigidly all-or-none. Generally, there is only one solution, which requires a long time to find. (d)-(e) By contrast, natural self-assembly (molecular or multicellular) consists of only a few types of identical components - schematized by one type in (d), two in (e), three in (f) - fitting each other tightly in tilings (e) or loosely in aggregates (d) and (f). This multiplicity and flexibility give rise to many possible approximate "solutions" via quick convergence times. Finally, no central process is steering the pieces. 


\subsection{From molecular self-assembly to multicellular self-assembly}

How, then, do self-assembling pieces find their way to their final positions on the basis of purely local interactions and create global order at the system level? At this point, principles of molecular self-assembly, on the one hand, and multicellular selfassembly, on the other hand, diverge in several important regards:

(iv) Existence of components: Molecules generally pre-exist in the solution before they self-assemble. Cells, however, are dynamically created during selfassembly by the division of other cells. ${ }^{1}$

(v) Binding fate: Molecules initially form a homogeneous mixture (the puzzle box) and bind to each other through stochastic collisions (possibly with help from enzymes, but the original encounter remains stochastic). Cells, however, appear on the spot, again by cellular division, in the neigborhood of the cells to which they bind (possibly later changing neighborhood through migration, but this is also a highly nonrandom process).

(vi) Shape determination: Possibly folding upon themselves after synthesis, molecules settle on a relatively fixed (passive) geometrical shape and admit only a limited amount of deformation when coming into contact with other molecules. Cells, however, dynamically and actively change their shape as they differentiate under the influence of molecular signalling from other cells (such as induction).

\subsubsection{Existence of components and binding fate}

The distinctions outlined in points (iv) and (v) are illustrated here with a simple model of swarm behavior (Fig. 2). In 2-D space, two types of particles, $\alpha$ and $\beta$ (respectively dark and light colored spots in the figure), interact via attractive and repulsive forces. By analogy with electric fields, these interactions are modeled as local energy potentials $V(\mathbf{r})$ that each particle emits in its vicinity as a function of vector $\mathbf{r}$ from its center. In this model, interaction potentials are the equivalent of the geometrical "shapes" of components, i.e., the specific binding affinities that they have with their neighbors. The two types of isotropic potentials, $V_{\alpha}(r)$ and $V_{\beta}(r)$, with $r=\|\mathbf{r}\|$, used in Fig. 2 are graphed in Fig. 3a,b. In both cases, they contain an impenetrable core of infinite values below $r_{c}$, representing the fact that particles are nondeformable discs of radius $r_{c} / 2$. At the other end of their interaction range, their "horizont" is defined by $r_{0}$. Beyond that distance, the energy landscape is flat and particles do not see one another. What distinguishes the two types is that an $\alpha$ particle is surrounded with a ring-shaped basin of attraction at some equilibrium distance $r_{e}<r_{0}$ (Fig. 3a), while a $\beta$ particle is not and simply repels other particles that come too close without attracting them (this virtually corresponds to $r_{0}<r_{e}$ ). The type- $\alpha$ basin is quadratic, simulating the establishment of a spring-like force with resting length $r_{e}$ as soon as two particles come closer than $r_{0}$ (but farther than $r_{c}$ ).

\footnotetext{
${ }^{1}$ Naturally, a new cell does not appear ex nihilo but is itself the result of self-assembly at a lower level of molecules synthesized by the mother cell, which draws pre-existing biochemical resources from the extracellular matrix. The present analogy, however, focuses on whole components and whether they are ready to assemble with other components at their own level.
} 
(b)
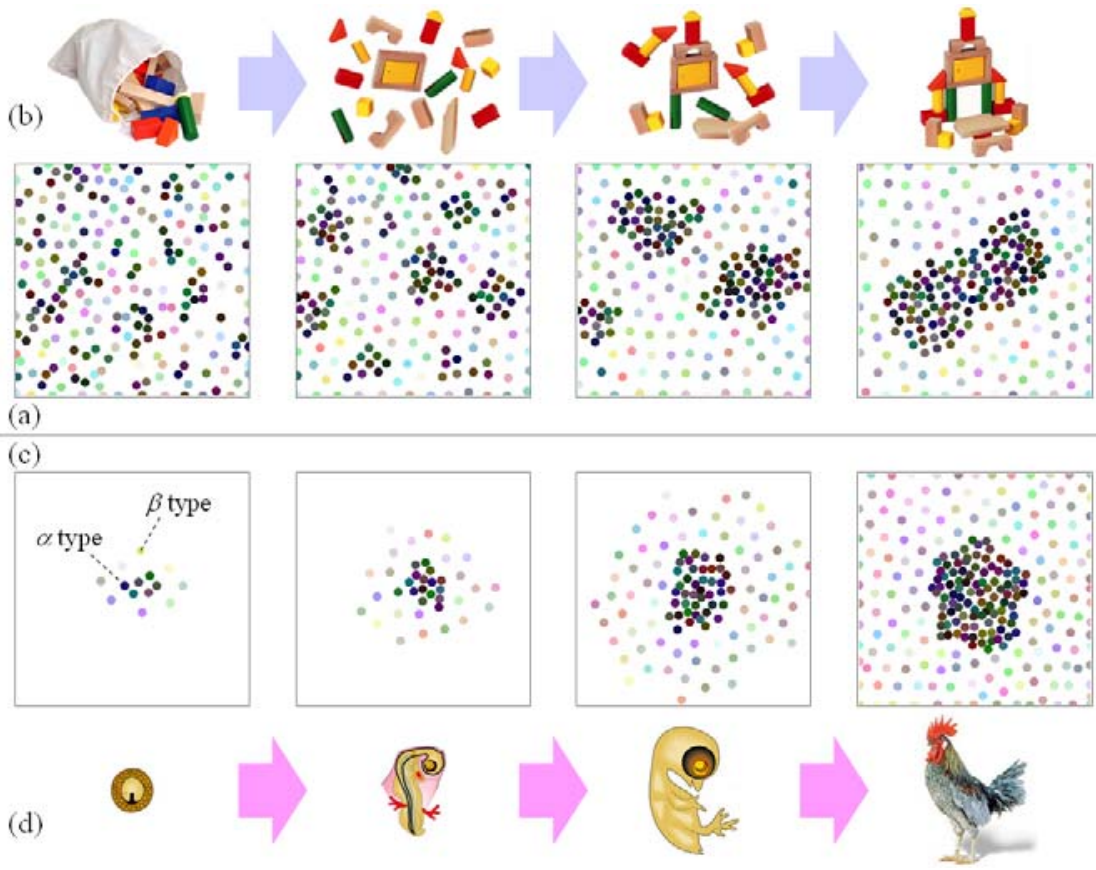

Fig. 2: Constrasting molecular-style self-assembly with multicellular-style self-assembly by a simple swarm model and metaphorical illustrations. The model contains particles of two types, $\alpha$ (dark color discs) and $\beta$ (light color discs), that exert an attractive and/or repulsive potential $V$ on their neighbors, with $V=V_{\alpha}$ for $\alpha-\alpha$ interactions (Fig. 3a with $r_{c}=1.6, r_{e}=3.5, r_{0}=5$ ) and $V=V_{\beta}$ for $\alpha-\beta$ and $\beta-\beta$ interactions (Fig. $3 b$ with $r_{c}=1.6, r_{0}=2.5$ ). Molecular selfassembly (a) relies on a random mix of pre-existing particles that sort out and aggregate through chance encounters. This would be equivalent to shaking a magnetic construction block game (b), in which pieces bear selective geometrical affinities. Multicellular self-assembly (c), however, as in animal development (d), mostly results from growth through cell division, not stochastic collisions. New cells are born already pre-positioned and rearrange only locally.

This swarming system is similar to previous models of collective motion in computer graphics [Reynolds 1987] and physics [Vicsek et al. 1995; see, e.g., Grégoire \& Chate 2004], with the difference that in those models particles are self-propelled at constant speed $v_{0}$ and only their direction of motion $\theta_{i}$ is updated at every time step. In the present model, the velocity may vary in both norm and direction according to a simplified equation of motion:

$$
\lambda \dot{\mathbf{x}}_{i}=-\sum_{j} \nabla_{i} V\left(\mathbf{x}_{j}, \mathbf{x}_{i}\right)+\eta
$$

where $\mathbf{x}_{i}$ is the position of particle $i, V\left(\mathbf{x}_{j}, \mathbf{x}_{i}\right)$ is the potential created by particle $j$ in $\mathbf{x}_{i}$, and $\eta$ is white noise. (The above equation can be derived from $m d^{2} \mathbf{x}_{i} / d t^{2}=$ $-\lambda d \mathbf{x}_{i} / d t-\sum_{j} \nabla_{i} V\left(\mathbf{x}_{j}, \mathbf{x}_{i}\right)+\eta$ by neglecting the inertia term $m \mathbf{x}^{\prime \prime}$ in front of the viscosity term $\lambda \mathbf{x}^{\prime}$, or assuming that particles are quasi-stationary.) Now, in the mixed type 
system of Fig. 2, $V\left(\mathbf{x}_{j}, \mathbf{x}_{i}\right)$ depends on both types of particles $i$ and $j$, such that $V\left(\mathbf{x}_{j}, \mathbf{x}_{i}\right)=V_{\alpha}\left(\mathrm{r}_{i j}\right)$, with $\mathbf{r}_{i j}=\left\|\mathbf{x}_{i}-\mathbf{x}_{j}\right\|$, if and only if $i$ and $j$ are of type $\alpha$; otherwise $V\left(\mathbf{x}_{j}, \mathbf{x}_{i}\right)=V_{\beta}\left(\mathrm{r}_{i j}\right)$ for the other type interactions $\alpha-\beta$ and $\beta-\beta$. This means that only $\alpha$ particles attract and lock in with other $\alpha$ particles, whereas they repel $\beta$ particles, which also repel each other. "Shape" is thus a relative concept for $\alpha$ particles, as they can switch between two affinity configurations depending on their neighbor's type.

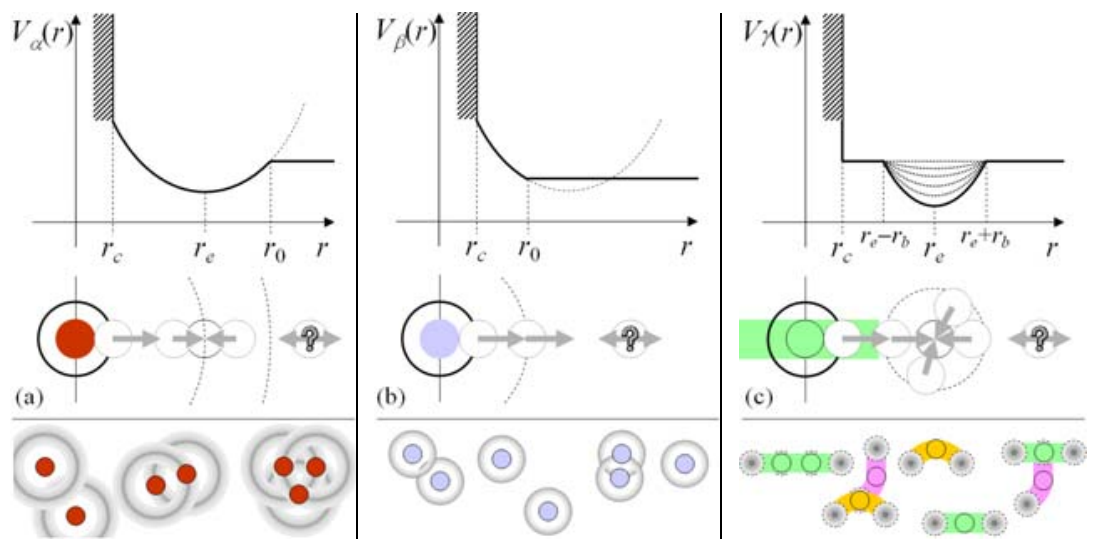

Fig. 3: The "shape" or binding affinities of self-assembling components can be modeled by the emission of local attraction/repulsion potentials $V$. In each frame: top-graph of $V$ as a function of distance $r$ from the particle's core; middle-2-D view "from above" of a neighbor particle's motion within $V$; bottom - example of a few particles interacting through $V$. (a) Isotropic elastic potential used in the $\alpha-\alpha$ interactions of Fig. 3. (b) Isotropic repulsion used in the $\alpha-\beta$ and $\beta-\beta$ interactions of Fig. 3. (c) Anisotropic "polar" attraction potential used in Fig. 4.

As a result, in Fig. 2a, an initial random mix of 110 particles of each type reliably converges toward a lower energy state: $\alpha$ particles collide by chance (random walk due to the stochastic term $\eta$ ), stick to one another and progressively form larger aggregates until a giant component containing all $\alpha$ particles is created in the midst of a sea of $\beta$ particles. Self-sorting processes such as this one have also been simulated using the Potts formalism [Graner \& Glazier 1992], a multivalued Ising model in which a pixel on the grid represents a fragment of a biological cell, and a local region of equal pixel values represents one cell. Instead of the point-wise motion of swarm systems, the Potts model shifts cell boundaries by flipping pixel values according to a stochastic surface energy minimization. Similarly to Eq. (1), Potts surface energy includes differential adhesion as a sum of pairwise interactions between cell types.

Although dissociated and mixed cells can spontaneously sort out again into homogeneous tissues, this phenomenon is seen mainly in artificial experiments whose goal is to demonstrate differences in cell adhesiveness. Cell sorting does not constitute a major natural developmental mechanism-despite common features with cell migration at the level of adhesion proteins. It might intervene locally as a correction mechanism (e.g., to compensate for small fluctuations or errors in cell differentiation) but, in the end, an organism does not emerge from a giant swarm of trillions of disag- 
gregated cells that reassemble in parallel. Biological morphogenesis is mostly the product of regulated growth, i.e., guided positioning by division and migration, not chance encounters.

For these reasons, Fig. 2a is a more faithful illustration of the molecular style of self-assembly (the first half of points (iv) and (v)), while multicellular-style selfassembly is better captured by Fig. $2 \mathrm{~b}$ (the second half of points (iv) and (v)). Here, the system starts out with only a few particles of each type, which later divide into same-type particles according to a certain probability. New particles pop up already pre-positioned near the type that produced them and only briefly rearrange within their local neighborhood. Not addressed in this simple model is cell migration, which also plays an important role in animal development. Yet again, migration has little to do with stochastic collisions. After its birth within a given neighborhood, a cell may traverse its environment toward a specific remote location, but it does so only under tight guidance from extracellular signals and cell-to-cell adhesion properties.

\subsubsection{Shape determination}

Once positioned, biological cells, unlike puzzle pieces or molecules, are also able to modify their individual shape dynamically and, consequently, the local geometrical arrangement that they form with their neighbors. This is an important aspect of multicellular self-assembly that was mentioned in point (vi) and will be modeled here by a variant of the previous swarm system. In this variant, illustrated in Fig. 3c and Fig. 4, the isotropic potentials $V_{\alpha}(r)$ and $V_{\beta}(r)$ are replaced with an anisotropic or "polar" potential $V_{\gamma}(\mathbf{r})$. Instead of $V_{\alpha}$ 's ring-shaped basin of attraction at distance $r_{e}$ from the cell center, the potential landscape $V_{\gamma}$ has two localized basins of attraction (quadratic wells of radius $\left.r_{b}\right)$ centered around two poles $\mathbf{r}_{1}=\left(\theta_{1}, r_{e}\right)$ and $\mathbf{r}_{2}=\left(\theta_{2}, r_{e}\right)$. For example, in Fig. $3 \mathrm{c}$ the values of $\theta_{1}$ and $\theta_{2}$ are 0 and $\pi$ in the case of the horizontal (green) segments. In Fig. 4, the swarm consists of $\gamma$ particles with "vertical" binding affinities $\theta_{1}=\pi / 2$ and $\theta_{2}=-\pi / 2$ (Fig. 4a,c) or variable angles (Fig. 4b,d). To represent the shape of this polar potential, these particles are displayed as short segments of length $r_{e}$ and thickness $r_{c}$, instead of discs. In Fig. 4a-b the particles pre-exist in a mix and have fixed shapes, whereas in Fig. 4c-d they dynamically appear and reshape themselves. Thus, while the constrast between Fig. 4a (colliding) and Fig. 4c (growing) reiterates points (iv) and (v) already illustrated in Fig. 2, here in the case of polar particles, the contrast between Fig. $4 \mathrm{~b}$ (fixed shapes) and Fig. 4d (dynamical shapes) focuses on point (vi). By dynamically changing their shape after placing themselves into chain formation (through colliding or growing), the particles of Fig. $4 \mathrm{~d}$ create a specific morphology, which is otherwise difficult or impossible to attain spontaneously through sheer stochastic encounters (Fig. 4b). The preshaped particles of Fig. $4 \mathrm{~b}$ have specific pairs of angles that replicate the final state of Fig. $4 \mathrm{~d}$ but are unable to coordinate; they only explore suboptimal and unstable states. By contrast, Fig. $4 \mathrm{~d}$ is analogous to the invagination of cell membranes, a common mechanism of animal development most striking during gastrulation, whereby a few cells constrict one of their sides (using filaments of motor proteins) and adopt an elongated "wedge" aspect that draws the entire sheet of neighbor cells towards them. 

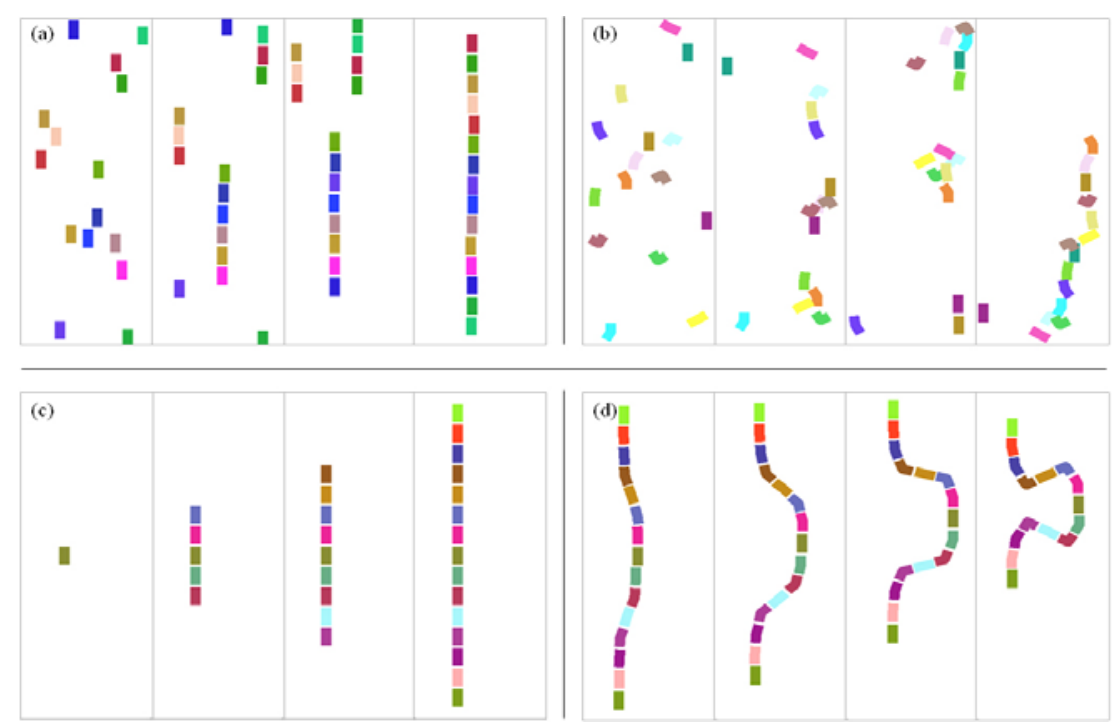

Fig. 4: Constrasting different modes of self-assembly in 1-D. In all frames, particles are of the same type $\gamma$, i.e., they interact via the polar potential $V_{\gamma}(r)$ of Fig. 3c. To remind of their anisotropic affinities, particles are drawn as small rectangles instead of discs. (a) Colliding selfassembly: 15 particles with vertical poles $\left(\theta_{1}, \theta_{2}\right)=(\pi / 2,-\pi / 2)$ quickly snap into place, forming a straight chain. (c) Growing self-assembly: as in Fig. 2c, the same string can be formed by dividing particles. (d) Reshaping self-assembly: each particle of (c) now dynamically bends its shape in specific ways (see Fig. 5c), making the string invaginate. (b) Preshaped self-assembly: the invagination cannot be reproduced by giving fixed angles to the particles in advance (the same angles that appear at the end of (d)) and letting them randomly collide.

In summary, biological self-assembly at the cellular level relies on principles that greatly facilitate and accelerate morphogenesis. When designing self-organized artificial systems in future, letting components dynamically create and reshape themselves "on the spot," as cells do, would be a far more efficient approach than letting them haphazardly try to match each other's pre-existing constraints, like molecules in a solution. (Obviously, a major technical difficulty will be to implement and control the self-replication of artificial components made of electric, chemical or even biological materials.) In any case, to transition from stochastic (molecular-style) self-assembly to programmable (multicellular-style) self-assembly, components must be able to modify their behavior (divide, differentiate, migrate) dynamically through communication. Cells do not just snap into place; they send molecular signals to each other, forming patterns of differentiation at the same time that they are self-assembling.

\section{Pattern Formation in Pre-Assembled Media}

Since Turing's 1952 seminal model of the spontaneous symmetry breaking and appearance of regular structures in biological organisms, the concept of morphogenesis 
has largely, but somewhat inaccurately, become synonymous with pattern formation. Morphogenesis originally referred to the biological development of the organs and structures of an organism during embryogenesis and, by extension of its etymology, any "generation of form" at various scales in other types of complex systems, such as physical (geomorphogenesis) or social (urban morphogenesis). Pattern formation, in contrast, generally refers to the emergence of statistically regular motifs in quasicontinuous and initially homogeneous 2-D or 3-D media. To be sure, both phenomena involve the decentralized self-organization of a myriad of elements and produce contrast where there was uniformity, yet they do not emphasize the same aspect of emerging order. The latter looks at shimmering landscapes of activity on a more or less fixed backdrop, while the former emphasizes the creation of intricate architectures and structures. Using an artistic metaphor, it could be said that pattern formation "paints" a pre-existing space, while morphogenesis "sculpts" its own space.

There is a huge diversity of pattern formation behaviors across many scales and substrates (e.g., fluid, electromagnetic, mechanical, chemical, biochemical), from which a few broad classes of mechanisms and models have been identified (e.g., convection cells, reaction-diffusion, activator-inhibitors, synchronization of oscillators). The observed patterns can be static, steady-state or dynamically changing (e.g., traveling waves) and organize themselves into patches or domains that also fall into a few classical geometrical families (e.g., spots, stripes, spirals, branches). Morever, the pattern formation processes typically studied are for the most part inherently stochastic, at both the microscopic level of elements and the macroscopic level of the distribution of patterns. Continuing the tradition initiated by Turing, most models have been focusing on systems that rely on instabilities and amplification of fluctuations to transition toward order and form patterns. Because of their randomness, and without carefully set boundary conditions (possibly themselves the product of morphogenesis; see below), the outcome of those processes is generally unpredictable in the number and position of the emerging mesoscopic domains (spots, stripes, convection cells). At the same time, the whole formation on the macroscopic level is fairly regular or even periodic, at least piecewise, since it essentially consists of repeated motifs. It displays statistical uniformity similar to textures.

In biological development, by contrast, the mesoscopic elements (organs, limbs, parts, tissues, etc.) are always very reliably positioned - unlike random spots and stripes - and display complex and heterogeneous morphologies - unlike uniform textures. Although the well-known colorful animal coats, such as seashell, zebrafish or leopard, have been (debatably) assumed to arise from morphogen-based reactiondiffusion pattern formation, they make up only a minor part of the whole organism. The unique characteristic of biological morphogenesis, absent from simpler physicalchemical pattern formation, is that each one of its self-organizing elements, the cell, contains a rich source of information stored in the DNA, which endows it with a vast repertoire of highly nontrivial behaviors. Even admitting that DNA is less than a "program," in the sense that it does not control the cell deterministically along a linear flow of execution, it is nonetheless, at the very least, a repository of stimuliresponse rules, vastly superior in quantity of functional information to any physical or chemical element involved in one of the habitual pattern formation dynamics. 
Embryogenesis, therefore, combines both pattern formation and morphogenetic self-assembly in a tightly integrated loop: It creates shape from patterning (Fig. 5) and patterns from shaping. Structures are "sculpted" from the assembly of elements that have been prompted to do so by the "painting" of their genetic identity. Conversely, newly formed shapes are able to support, and trigger, new domains of genetic expression. DNA is "consulted" at every step of this exchange, in every cell, to produce the proteins that will guide the cell's highly specific biomechanic behavior (shaping) and signalling behavior (patterning) at a given time in a given location, depending on the signals received from the neighborhood. A schematic illustration of the "shape from patterning" process is shown in Fig. 5.

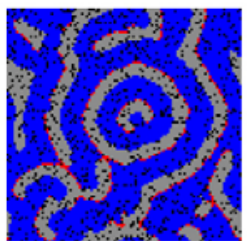

(a)

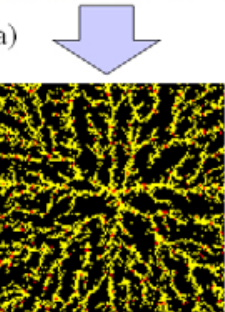

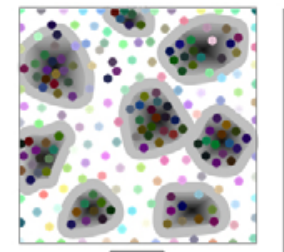

(b)

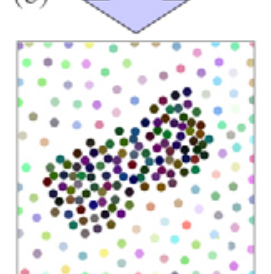

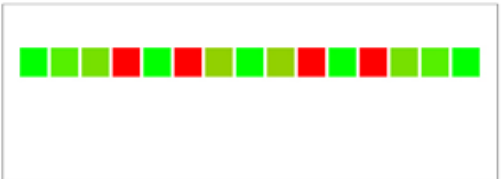

(c)

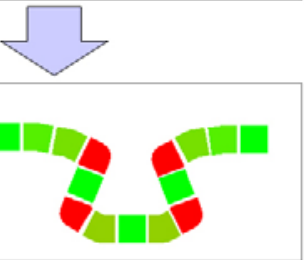

Fig. 5: "Shape from patterning" examples: deriving morphogenetic self-assembly (bottom frames) from pattern formation (top frames). (a) Amoebae in the slime mold Dictyostelium first generate waves of chemical signalling, modeled as a lattice of coupled oscillators (top). After a while, the lattice breaks up as cells follow the concentration gradient toward wave centers and aggregate (bottom). (snapshots from T. Schmickl's online simulations at http://zool33.unigraz.at/schmickl) (b) Augmented view of the swarm of Fig. 2a, where the $\alpha$ particles are assumed to have differentiated from a prepattern of chemical concentration before assembling as above. (c) Augmented view of the chain of Fig. $4 \mathrm{~d}$, in which the bending angle of each cell is also determined by a prepattern of identity. (The type of pattern formation in (b) and (c), whether Turing-like, genetically regulated or a combination of the two, is not specified here.)

\section{Integrating Self-Assembly and Pattern Formation Under Genetic Regulation}

The model of artificial embryogenesis that I have recently proposed [Doursat 2006, 2007] is an original attempt to integrate the three fundamental ingredients discussed above: (i) self-assembly (SA) and (ii) pattern formation (PF), triggering each other in a feedback loop under the tight control of (iii) nonrandom genetic instructions (GI) here, a gene regulatory network - stored in each cell of the system. Previous theoretical models of biological development or bio-inspired artificial life systems have sel- 
dom included all three mechanisms. The evo-devo works of Hogeweg [2000], Salazar-Ciudad \& Jernvall [2002] or, with lesser morphogenetic abilities, the Cellerator system [Shapiro et al. 2003] and Nagpal's origami [2002] are a few notable achievements. Other interesting studies have explored the combination of two out of three ingredients: SA and PF, no GI-self-assembly based on cell adhesion and signalling pattern formation, but using only predefined cell types without internal genetic variables [e.g., Marée \& Hogeweg 2001]; PF and GI, no SA - nontrivial pattern formation from instruction-driven intercellular signalling, but on a fixed lattice without self-assembling motion [e.g., von Dassow et al. 2000, Coore 1999]; SA and GI, no $\mathrm{PF}$ - heterogeneous swarms of genetically programmed, self-assembling particles, but in empty space without mutual differentation signals [e.g., Sayama 2007].

The present model has been explained in detail elsewhere [Doursat 2006, 2007]. It is summarized here, highlighting the interplay between pattern formation and selfassembly, as illustrated in Fig. 6.

\subsection{Gene-regulated pattern formation}

A virtual embryo is a swarm of cells, where each cell contains a gene regulatory network (GRN) coding for its signalling and mechanic activity. Through intercellular coupling between neighboring GRNs, the embryo becomes patterned into identity domains of differentiated gene expression, creating a "hidden geography" revealed by in situ hybridization (Fig. 6c,e). Essentially, logical combinations of regulatory switches (OR, AND) translate into geometric combinations of precursor patterns into new patterns (union, intersection). Developmental genes are roughly organized in tiers, or "generations." Earlier genes map the way for later genes and gene expression propagates in a cascade. This principle has been beautifully demonstrated in the Drosophila embryo. The intersection of various striping patterns along its three main axes gives rise to smaller domains such as the organ primordia and "imaginal discs," which are groups of cells marking the location and identity of the fly's future appendages (legs, wings, antennae). Going back in time, the whole process begins with concentration gradients of maternal proteins diffusing across the initial cluster of cells. These gradients are the functional equivalent of a coordinate system.

\subsection{Biomechanic self-assembly}

In parallel to genetic patterning, the embryo continues to grow and undergo extensive reshaping as cells divide and proliferate. Previous identity domains expand and deform while becoming partitioned into new and finer identity domains. Three main biomechanic principles responsible for these morphogenetic changes are integrated into the model as schematic rules: (a) differential adhesion as elastic forces between cells, (b) inhomogeneous cell division as internal probability rates, and (c) tropic cell migration as internal velocity vectors. In parallel to chemical coupling between their GRNs, neighboring cells are connected by abstract mechanical edges between their nuclei, established through Delaunay triangulation - the cell shapes being the complementary Voronoi domains. Similarly to the $V_{\alpha}(r)$ potential of Fig. 3a, cell-to-cell edges are modeled as small springs, so that each cell tries to set the distance with its 
neighbors' nuclei to $r_{e}$. Biological cells also stick to one another by means of adhesion proteins that cover their membrane. The great diversity of surface adhesion proteins gives them the ability to selectively recognize one another by modulating their degree of "stickiness." In the elastic force model, differential adhesion on edge $i \leftrightarrow j$ between cells $i$ and $j$ is modeled by resting lengths $l_{i j}$ spring coefficients $k_{i j}$ that can vary from edge to edge. Different proliferation frequencies $\rho_{i}$ also create deformation in the embryo, as compartments expand faster than others. Migration, represented by additional time- and space-varying vectors $\mathbf{v}_{i}$, is not shown in this article.

\subsection{Pattern-regulated self-assembly, assembly-triggered patterns}

Closing the loop, the complete model establishes a functional dependency between cell identities and mechanical cell behaviors (Fig. 6). The self-assembly rules of section 3.2 are linked to the self-patterning process of section 3.1 by making mechanical parameters $\rho_{i}, l_{i j}$ and $k_{i j}$ depend on the current state of the genetic expression of $i$ and $j$, i.e., the identity (colored) domains to which cells belong. See Fig. 6 for details.

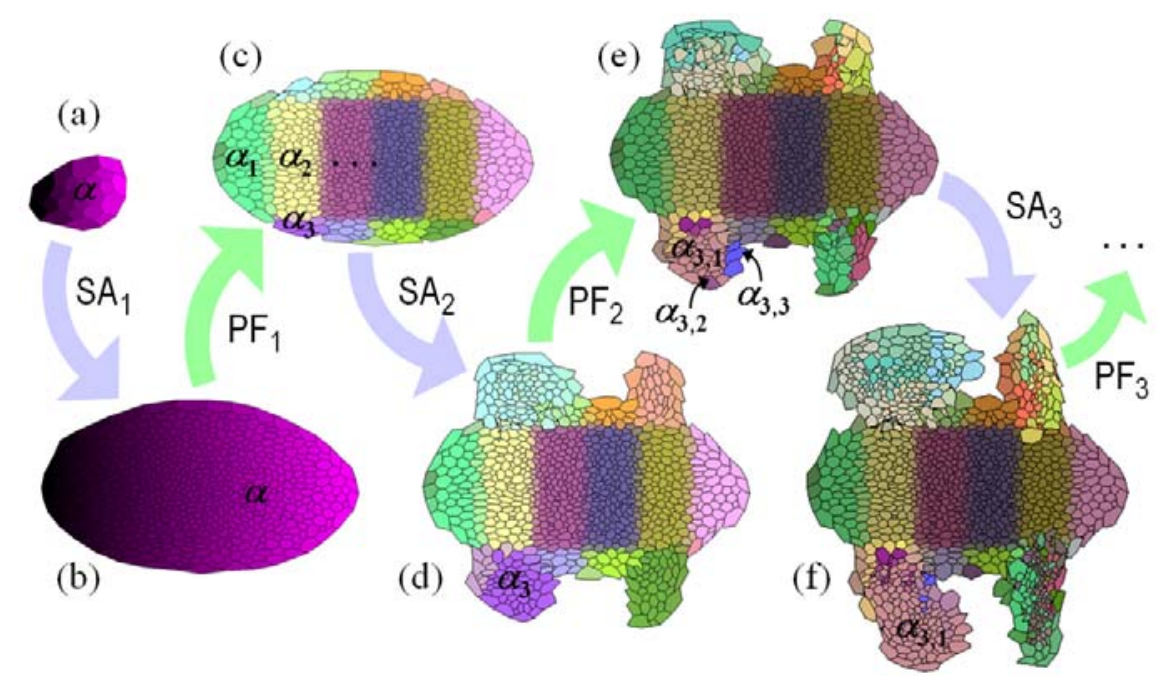

Fig. 6: Integrating self-assembly (SA) and pattern formation (PF) under genetic regulation. (a) Starting from a small clump, cells proliferate at a uniform division rate to reach about 800 . All cells are of same type $\alpha$ with an anisotropic 2:1 ratio along $x$ (ellipsoid version of elastic potential $V_{\alpha}$, Fig. 3a). (b) As in Fig. 2b, spontaneous rearrangements give the embryo a convex shape, here oval. During expansion, protein gradients ( $x$-gradient in purple) spread across the unique domain. (c) The varying concentration levels are then read out by each cell and input into the first stages of their GRNs (not shown), producing in output different values of gene expression, i.e., defining new cell types $\alpha_{1}, \alpha_{2}$ and so on. (d) These types, in turn, determine the new division rates $\rho(\alpha)$ in each domain and adhesion coefficients $k\left(\alpha, \alpha^{\prime}\right)$ between domains. For example, $\alpha_{3}$ and three other types start proliferating at a faster rate than the rest of the embryo, while they lose adhesion with neighboring domains, thereby creating limb-like bulges. (e)-(f) The same alternation of PF-induced differentiation and heterogenous-type SA continues at a finer scale of detail with respect to the overall expanding organism. 


\section{Toward Evolutionary Meta-Design}

Exploding growth in hardware components, software modules and network users will force us to find an alternative to rigidly designing and controlling computational systems in every detail. Instead, future progress in information and communication technologies will depend on our ability to meta-design mechanisms allowing those systems to self-assemble, self-regulate and evolve. Nature offers a great variety of decentralized, autonomous systems, most conspicuously biological organisms. Deemed "complex," these systems might in fact be less costly, more efficient or even "simpler" than human-designed and centrally controlled contrivances. Complex systems are characterized by the self-organization of a great number of small, repeated elements into large-scale, adaptive patterns, where each element may itself obey the dynamics of an inner network of smaller entities at a finer scale (microprogram). The new engineering challenge is thus to "guide" this self-organization, i.e., to prepare the conditions and mechanisms favorable to nonrandom, heterogeneous and reproducible morphogenesis (macro-program). At the same time, it is also to allow the parameters of this process evolve in order to freely generate innovative designs. Finding efficient systems will require matching loose selection criteria with productive variation mechanisms. The first point concerns the openness of the designers to "surprising" outcomes; the second point concerns the intrinsic ability of complex systems to create a "solution-rich" space [Minai et al. 2006] by combinatorial tinkering on highly redundant parts. Embryogenesis, the development of an entire organism from a single cell, provides the most striking example of self-organization guided by evolvable genetic information.

This work describes an original model of bio-insipred, artificial embryomorphic system growth, integrating pattern formation and self-assembly under non-random genetic regulation. A virtual organism is represented by a mass of cells that proliferate, migrate and self-pattern into differentiated domains. Each cell contains an internal gene regulatory network and acquires a specific gene expression identity by interaction with neighboring cells. Differentiated cell types trigger different cell behaviors, which in turn induce new identities. The organism's final architecture depends on the detailed interplay between the various rates of cell division and movement, propagation of genetic expression and positional information. Ultimately, on this score of "theme and variations" (developmental laws and parameters), evolution will be the player.

Based on these first results, I propose a new discipline, embryomorphic engineering as a "fine-grain" approach to systems design, based on swarms of relatively simple, cloned elements. It emphasizes the need for hyper-distributed architectures and self-organized development as prerequisites for evolutionary innovation. In possible future hardware applications, nano-units containing the same instructions could selforganize without the need for reliability or precise arrangement as in traditional VLSI [Coore 1999, Nagpal 2002]. In software or network applications (servers, security, etc.), a swarm of small-footprint software agents could diversify and self-deploy to achieve a desired level of functionality. 


\section{Bibliography}

[1] Coore, D., 1999, Botanical Computing: A Developmental Approach to Generating Interconnect Topologies on an Amorphous Computer, Ph.D. thesis, MIT Department of Electrical Engineering and Computer Science.

[2] Doursat, R., 2006, The growing canvas of biological development: Multiscale pattern generation on an expanding lattice of gene regulatory networks, InterJournal: Complex Systems, 1809.

[3] Doursat, R., 2007, Organically grown architectures: Creating decentralized, autonomous systems by embryomorphic engineering, In Organic Computing, R. P. Würtz, ed., Springer-Verlag, to appear.

[4] Graner, F., \& Glazier, J. A., 1992, Simulation of biological cell sorting using a 2-D extended Potts model, Physical Review Letters, 69(13): 2013-2016.

[5] Grégoire, G., \& Chaté, H., 2004, Onset of collective and cohesive motion, Physical Review Letters, 92: 025702.

[6] Hogeweg, P., 2000, Evolving mechanisms of morphogenesis: On the interplay between differential adhesion and cell differentiation, Journal of Theoretical Biology, 203: 317-333.

[7] Marée, A. F. M., \& Hogeweg, P., 2001, How amoeboids self-organize into a fruiting body: Multicellular coordination in Dictyostelium discoideum, PNAS, 98(7): 3879-3883.

[8] Minai, A. A., Braha, D., \& Bar-Yam, Y., 2006, Complex engineered systems, in Complex Engineered Systems: Science Meets Technology, D. Braha, Y. BarYam and A. A. Minai, eds., Springer-Verlag.

[9] Nagpal, R., 2002, Programmable self-assembly using biologically-inspired multi-agent control, 1st Int Conf on Autonomous Agents, Bologna, July 15-19.

[10] Reynolds, C. W., 1987, Flocks, herds and schools: A distributed behavioral model. Computer Graphics, 21(4): 25-34.

[11] Salazar-Ciudad, I., \& Jernvall, J., 2002, A gene network model accounting for development and evolution of mammalian teeth, PNAS, 99(12), 8116-8120.

[12] Sayama, H., 2007, Decentralized control and interactive design methods for large-scale heterogeneous self-organizing swarms, Advances in Artificial Life: Proceedings of the 9th European Conference on Artificial Life (ECAL).

[13] Shapiro, B. E., Levchenko, A., Meyerowitz, E. M., Wold, B. J., \& Mjolsness, E. D., 2003, Cellerator: Extending a computer algebra system to include biochemical arrows for signal transduction simulations, Bioinf, 19(5): 677-678.

[14] Vicsek, T., Czirók, A., Ben-Jacob, E., Cohen, I., \& Shochet, O., 1995, Novel type of phase transition in a system of self-driven particles, Physical Review Letters, 75: 1226-1229.

[15] von Dassow, G., Meir, E., Munro, E. M., \& Odell, G. M., 2000, The segment polarity network is a robust developmental module. Nature 406: 188-192. 